\title{
Instantons on the six-sphere and twistors
}

Olaf Lechtenfeld, and Alexander D. Popov

Citation: Journal of Mathematical Physics 53, 123506 (2012);

View online: https://doi.org/10.1063/1.4765065

View Table of Contents: http://aip.scitation.org/toc/jmp/53/12

Published by the American Institute of Physics

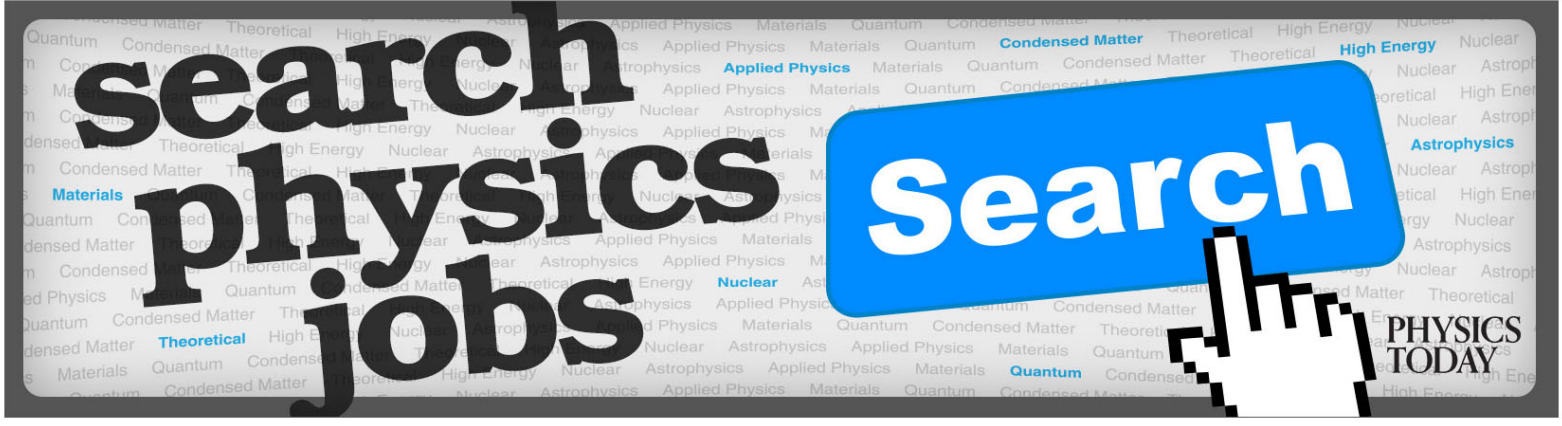




\title{
Instantons on the six-sphere and twistors
}

\author{
Olaf Lechtenfeld ${ }^{1,2, a)}$ and Alexander D. Popov ${ }^{3, b)}$ \\ ${ }^{1}$ Institut für Theoretische Physik and Riemann Center for Geometry and Physics, \\ Leibniz Universität Hannover, Appelstraße 2, 30167 Hannover, Germany \\ ${ }^{2}$ Centre for Quantum Engineering and Space-Time Research, Leibniz Universität Hannover, \\ Welfengarten 1, 30167 Hannover, Germany \\ ${ }^{3}$ Bogoliubov Laboratory of Theoretical Physics, JINR, 141980 Dubna, \\ Moscow Region, Russia
}

(Received 25 June 2012; accepted 12 October 2012; published online 8 November 2012)

We consider the six-sphere $S^{6}=G_{2} / \mathrm{SU}(3)$ and its twistor space $\mathcal{Z}=G_{2} / \mathrm{U}(2)$ associated with the $\mathrm{SU}(3)$-structure on $S^{6}$. It is shown that a Hermitian Yang-Mills connection (instanton) on a smooth vector bundle over $S^{6}$ is equivalent to a flat partial connection on a vector bundle over the twistor space $\mathcal{Z}$. The relation with Tian's tangent instantons on $\mathbb{R}^{7}$ and their twistor description are briefly discussed. (c) 2012 American Institute of Physics. [http://dx.doi.org/10.1063/1.4765065]

\section{INTRODUCTION AND SUMMARY}

The twistor description of solutions to chiral zero-rest-mass field equations on the sixdimensional space $\mathbb{C}^{6}$ or its real forms with various signatures (see, e.g., Ref. 1) was generalized recently to Abelian ${ }^{2,3}$ and non-Abelian ${ }^{4}$ holomorphic principal 2-bundles over the twistor space $Q_{6}^{\prime} \subset \mathbb{C} P^{7} \backslash \mathbb{C} P^{3}$, corresponding to solutions of the 3 -form self-duality equations on $\mathbb{C}^{6}$. The twistor approach was also extended to maximally supersymmetric Yang-Mills theory on $\mathbb{C}^{6} .{ }^{5}$ Twistor methods have also been applied in the study of scattering amplitudes in this theory (see, e.g., Ref. 6).

The goal of our paper is to describe instantons in gauge theory on Euclidean six-dimensional space (i.e., the bosonic sector of maximally supersymmetric Yang-Mills theory) by using twistor methods. Recall that instantons in four dimensions are nonperturbative gauge-field configurations solving conformally invariant first-order anti-self-duality equations, which imply the full Yang-Mills equations. ${ }^{7}$ The twistor approach allows one to describe instanton solutions and their moduli space very efficiently. ${ }^{8-10}$ We will apply it to study gauge instantons on the six-dimensional sphere $S^{6}$, which is a natural compactification of $\mathbb{R}^{6}$. Our considerations are based on papers studying twistor spaces associated with higher-dimensional manifolds ${ }^{11-19}$ as well as on papers considering instanton equations in dimensions higher than four. ${ }^{20-29}$ Here, we consider instanton equations only on $S^{6}$. However, our results can be generalized to any nearly Kähler manifold in six and higher dimensions as well as to some other manifolds with $G$-structure.

We recall some definitions to clarify our purposes. Let $X$ be a Riemannian manifold of dimension $2 n$. We define the metric twistor space of $X$ as the bundle $\operatorname{Tw}(X) \rightarrow X$ of almost Hermitian structures on $X$ (i.e., almost complex structures compatible with the metric $g$ on $X$ and its orientation) associated with the principal bundle $P(X, \mathrm{SO}(2 n))$ of orthonormal frames of $X$, i.e.,

$$
\operatorname{Tw}(X):=P(X, \mathrm{SO}(2 n)) \times_{\mathrm{SO}(2 n)} \mathrm{SO}(2 n) / \mathrm{U}(n) .
$$

It is well known that $\operatorname{Tw}(X)$ can be endowed with an almost complex structure $\mathcal{J}$, which is integrable if and only if the Weyl tensor of $X$ vanishes identically when $n>2 .{ }^{11}$ In the case $n=2$, the Weyl tensor has to be anti-self-dual. ${ }^{9,30}$ However, if the manifold $X$ has a $G$-structure (which is

\footnotetext{
a)E-mail: lechtenf@itp.uni-hannover.de.

b)E-mail: popov@theor.jinr.ru.
} 
not necessarily integrable) then one can often find a subbundle $\mathcal{Z}$ of $\operatorname{Tw}(X)$ associated with the $G$-structure bundle $P(X, G)$ for $G \subset \mathrm{SO}(2 n)$, such that an induced almost complex structure (also called $\mathcal{J}$ ) on $\mathcal{Z}$ is integrable. Many examples were considered in the literature. ${ }^{11-13,15-17}$

The six-sphere $S^{6}$ provides an interesting example. Considered as the round sphere

$$
S^{6}=\operatorname{Spin}(7) / \operatorname{Spin}(6) \text {, }
$$

its metric twistor space is

$$
\operatorname{Tw}\left(S^{6}\right)=\operatorname{Spin}(7) / \mathrm{U}(3) \stackrel{\mathbb{C} P^{3}}{\longrightarrow} S^{6},
$$

(see, e.g., Refs. 14 and 17), which may be recognized as a six-dimensional quadric ${ }^{38} Q_{6}$ in $\mathbb{C} P^{7}$. Alternatively, one may consider the six-sphere as a nearly Kähler homogeneous space with SU(3)structure, namely

$$
S^{6}=G_{2} / \mathrm{SU}(3)
$$

Then,

$$
\mathcal{Z}=G_{2} / \mathrm{U}(2) \stackrel{\mathbb{C} P^{2}}{\longrightarrow} S^{6}
$$

is a complex subbundle of $\operatorname{Tw}\left(S^{6}\right) \cdot{ }^{11-13,16}$ Note that $\mathcal{Z}$ can be identified with a five-dimensional quadric $Q_{5} \subset \mathbb{C} P^{6}$, and obviously $Q_{5} \subset Q_{6}=\mathrm{Tw}\left(S^{6}\right)$. The twistor space (1.5) is a bundle of almost complex structures $J$ on $S^{6}$, which are parametrized by the complex projective space $\mathbb{C} P^{2}$ at each point of $S^{6}$.

There was an attempt, ${ }^{31}$ not quite successful, to obtain instanton-type configurations on $S^{6}$ from holomorphic bundles over $\operatorname{Tw}\left(S^{6}\right)$. However, natural instanton equations on $S^{6}$ (as well as on $\mathbb{R}^{6}$ ) are the Donaldson-Uhlenbeck-Yau (DUY) equations, ${ }^{21,39}$ which are SU(3) invariant but not invariant under the $\mathrm{SO}(6)$ transformations on the round six-sphere.

The DUY equations are well defined on $S^{6}=G_{2} / \mathrm{SU}(3)$, and their solutions are natural connections $\mathcal{A}$ on pseudo-holomorphic vector bundles $E \rightarrow S^{6} .{ }^{32}$ We will show that such bundles $(E, \mathcal{A})$ are pulled back to complex vector bundles $(\widetilde{E}, \widetilde{\mathcal{A}})$ over the complex twistor space $\mathcal{Z}=G_{2} / \mathrm{U}(2)$ with flat partial connection $\widetilde{\mathcal{A}}$. For the definition and discussion of such connections, see, e.g., Refs. 19,33 , and 34. The bundle $\widetilde{E} \rightarrow \mathcal{Z}$ is not holomorphic. We would like to emphasize two outcomes of our study of instantons on $S^{6}$ :

(i) the reduced twistor space $\mathcal{Z} \hookrightarrow \operatorname{Tw}(X)$ of $X$ may be more suitable for describing solutions of field equations on manifolds $X$ with $G$-structure than the metric twistor space $\operatorname{Tw}(X)$,

(ii) the twistor description of gauge instantons in dimensions higher than four may lead to nonholomorphic bundles over the reduced twistor space $\mathcal{Z}$ even if $\mathcal{Z}$ is a complex manifold.

\section{NEARLY KÄHLER STRUCTURE ON $\boldsymbol{S}^{6}$}

\section{A. Almost complex structure}

Let us consider the principal fibre bundle

$$
G_{2} \longrightarrow G_{2} / \mathrm{SU}(3)=S^{6}
$$

with the Lie group SU(3) as the structure group. Let $\left\{e^{a}\right\}$ with $a=1, \ldots, 6$ be a (local) coframe on $S^{6}$ compatible with the SU(3)-structure and $\left\{e^{i}\right\}$ with $i=7, \ldots, 14$ be the components of an $s u(3)$ valued connection on the bundle (2.1). Using $e^{a}$, one can introduce an almost complex structure $J$ on $S^{6}$ such that

$$
J \theta^{\alpha}=\mathrm{i} \theta^{\alpha}, \alpha=1,2,3, \quad \text { for } \theta^{1}:=e^{1}+\mathrm{i} e^{2}, \theta^{2}:=e^{3}+\mathrm{i} e^{4}, \theta^{3}:=e^{5}+\mathrm{i} e^{6},
$$

as well as define forms

$$
\omega:=\frac{\mathrm{i}}{2}\left(\theta^{1} \wedge \theta^{\overline{1}}+\theta^{2} \wedge \theta^{\overline{2}}+\theta^{3} \wedge \theta^{\overline{3}}\right) \quad \text { and } \quad \Omega:=\theta^{1} \wedge \theta^{2} \wedge \theta^{3} .
$$




\section{B. Flat connection on $S^{6}$}

It is convenient to work with the matrices

$$
\begin{gathered}
\theta:=\varkappa\left(\theta^{1} \theta^{2} \theta^{3}\right), \quad \bar{\theta}:=\varkappa\left(\theta^{\overline{1}} \theta^{\overline{2}} \theta^{\overline{3}}\right), \\
B:=\sigma\left(\begin{array}{ccc}
0 & \theta^{3} & -\theta^{2} \\
-\theta^{3} & 0 & \theta^{1} \\
\theta^{2} & -\theta^{1} & 0
\end{array}\right) \quad \text { and } \quad \bar{B}:=\sigma\left(\begin{array}{ccc}
0 & \theta^{\overline{3}} & -\theta^{\overline{2}} \\
-\theta^{\overline{3}} & 0 & \theta^{\overline{1}} \\
\theta^{\overline{2}} & -\theta^{\overline{1}} & 0
\end{array}\right)
\end{gathered}
$$

with $\varkappa=\sqrt{\frac{2}{3}}$ and $\sigma=\sqrt{\frac{1}{3}}$. Using (2.4) and (2.5), one can introduce a flat Lie $G_{2}$-valued connection $\mathcal{A}_{0}{ }^{35}$ on the trivial bundle $G_{2} \times S^{6} \rightarrow S^{6}$ as

$$
\mathcal{A}_{0}=\left(\begin{array}{ccc}
\bar{\Gamma} & -\theta^{\dagger} & B \\
\theta & 0 & \bar{\theta} \\
\bar{B} & -\bar{\theta}^{\dagger} & \Gamma
\end{array}\right) \quad \text { with } \quad \Gamma=e^{i} I_{i} \quad \text { and } \quad \bar{\Gamma}=e^{i} \bar{I}_{i}=-e^{i} I_{i}^{\top} \text {, }
$$

where $I_{i}=-I_{i}^{\dagger}$ are $3 \times 3$ matrix generators of the group $\mathrm{SU}(3)$ and $\Gamma=e^{i} I_{i}$ is the canonical connection in the bundle (2.1).

\section{Maurer-Cartan equations on $\boldsymbol{S}^{6}$}

The flatness of the connection (2.6) (see Ref. 35) means that there exists a local $G_{2}$-valued function $L$, which is a coset representative of $G_{2} / \mathrm{SU}(3)$ such that $\mathcal{A}_{0}=L^{-1} \mathrm{~d} L$. Note that $L$ is a local section of the bundle (2.1). For the curvature $\mathcal{F}_{0}=\mathrm{d} \mathcal{A}_{0}+\mathcal{A}_{0} \wedge \mathcal{A}_{0}$, we find

$$
\mathcal{F}_{0}=\left(\begin{array}{ccc}
\bar{R}-\theta^{\dagger} \wedge \theta+B \wedge \bar{B} & -\left(\mathrm{d} \theta^{\dagger}+\bar{\Gamma} \wedge \theta^{\dagger}+B \wedge \bar{\theta}^{\dagger}\right) & \mathrm{d} B+\bar{\Gamma} \wedge B-\theta^{\dagger} \wedge \bar{\theta}+B \wedge \Gamma \\
\mathrm{d} \theta+\theta \wedge \bar{\Gamma}+\bar{\theta} \wedge \bar{B} & -\left(\theta \wedge \theta^{\dagger}+\bar{\theta} \wedge \bar{\theta}^{\dagger}\right) & \mathrm{d} \bar{\theta}+\bar{\theta} \wedge \Gamma+\theta \wedge B \\
\mathrm{~d} \bar{B}+\bar{B} \wedge \bar{\Gamma}-\bar{\theta}^{\dagger} \wedge \theta+\Gamma \wedge \bar{B} & -\left(\mathrm{d} \bar{\theta}^{\dagger}+\Gamma \wedge \bar{\theta}^{\dagger}+\bar{B} \wedge \theta^{\dagger}\right) & R-\bar{\theta} \wedge \bar{\theta}+\bar{B} \wedge B
\end{array}\right) .
$$

From $\mathcal{F}_{0}=0$, it follows that

$$
\mathrm{d}\left(\begin{array}{l}
\theta^{1} \\
\theta^{2} \\
\theta^{3}
\end{array}\right)+\Gamma \wedge\left(\begin{array}{l}
\theta^{1} \\
\theta^{2} \\
\theta^{3}
\end{array}\right)=\frac{2}{\sqrt{3}}\left(\begin{array}{c}
\theta^{\overline{2}} \wedge \theta^{\overline{3}} \\
\theta^{\overline{3}} \wedge \theta^{\overline{1}} \\
\theta^{\overline{1}} \wedge \theta^{\overline{2}}
\end{array}\right) \quad \Rightarrow \quad \mathrm{d} \theta^{\alpha}+\Gamma_{\beta}^{\alpha} \wedge \theta^{\beta}=T^{\alpha}
$$

where $\Gamma=\left(\Gamma_{\beta}^{\alpha}\right)=\left(\Gamma^{i} I_{i \beta}^{\alpha}\right)$ is the canonical connection on the tangent bundle $T S^{6}$ associated to the bundle (2.1), and where $T^{\alpha}=\frac{1}{2} T_{\bar{\beta} \bar{\gamma}}^{\alpha} \theta^{\bar{\beta}} \wedge \theta^{\bar{\gamma}}$ is the intrinsic torsion of $\Gamma$ (see, e.g., Ref. 36). Equation (2.8) and its complex conjugate constitute the Maurer-Cartan equations on the sphere $S^{6}$.

The curvature $R=\mathrm{d} \Gamma+\Gamma \wedge \Gamma$ of the connection $\Gamma$ is read off (2.7) by equating to zero its lower right (or upper left) block,

$$
R=\bar{\theta}^{\dagger} \wedge \bar{\theta}-\bar{B} \wedge B=\frac{1}{3}\left(\begin{array}{ccc}
2 \theta^{1 \overline{1}}-\theta^{2 \overline{2}}-\theta^{3 \overline{3}} & 3 \theta^{1 \overline{2}} & 3 \theta^{1 \overline{3}} \\
3 \theta^{2 \overline{1}} & -\theta^{1 \overline{1}}+2 \theta^{2 \overline{2}}-\theta^{3 \overline{3}} & 3 \theta^{2 \overline{3}} \\
3 \theta^{3 \overline{1}} & 3 \theta^{3 \overline{2}} & -\theta^{1 \overline{1}}-\theta^{2 \overline{2}}+2 \theta^{3 \overline{3}}
\end{array}\right)
$$

where $\theta^{1 \overline{1}}=\theta^{1} \wedge \theta^{\overline{1}}$ etc. Also, from (2.8) we see that the almost complex structure (2.2) on $S^{6}$ is not integrable due to the torsion $T^{\alpha}$, which is a $(0,2)$-form with respect to $J$. It is easy to show that

$$
\mathrm{d} \omega=3 \rho \operatorname{Im} \Omega \quad \text { and } \quad \mathrm{d} \Omega=2 \rho \omega \wedge \omega,
$$

where $\rho \in \mathbb{R}$ is proportional to the inverse radius of $S^{6}$. The pair $(\omega, \Omega)$ of forms subject to (2.10) turns $S^{6}$ into a nearly Kähler manifold (see, e.g., Refs. 16,32, and 36). It comes with a non-integrable $\mathrm{SU}(3)$-structure. 


\section{Hermitian Yang-Mills equations}

Consider an oriented $2 n$-dimensional Riemannian manifold $X^{2 n}$ with an almost complex structure $J$ and a complex vector bundle $E$ over $X^{2 n}$ with a connection $\mathcal{A}$. According to Bryant, ${ }^{32}$ a connection $\mathcal{A}$ on $E$ defines a pseudo-holomorphic structure if it has curvature $\mathcal{F}=\mathrm{d} \mathcal{A}+\mathcal{A} \wedge \mathcal{A}$ of type $(1,1)$ with respect to $J$, i.e., if $\mathcal{F}^{0,2}=0=\mathcal{F}^{2,0}$.

One can endow the bundle $E$ with a Hermitian metric and choose $\mathcal{A}$ to be compatible with the Hermitian structure on $E$. If, in addition, $\omega$ is an almost Hermitian structure on $\left(X^{2 n}, J\right)$ and $c_{1}(E)$ $=0,{ }^{40}$ then the equations

$$
\left.\mathcal{F}^{0,2}=-\left(\mathcal{F}^{2,0}\right)^{\dagger}=0 \quad \text { and } \quad \omega\right\lrcorner \mathcal{F}:=\omega^{a b} \mathcal{F}_{a b}=0
$$

are called the Hermitian Yang-Mills equations. The notation $\omega\lrcorner$ exploits the underlying Riemannian metric $g=\delta_{a b} e^{a} e^{b}$. In the case of an integrable almost complex structure $J$ on $X^{2 n}$, these equations were introduced by Donaldson and Uhlenbeck and Yau. ${ }^{21}$

We notice that the canonical connection $\Gamma$ on the tangent bundle of $S^{6}=G_{2} / \mathrm{SU}(3)$ satisfies the DUY Equation (2.11). In other words, its curvature obeys $R^{2,0}=0=R^{0,2}$ and $\left.\omega\right\lrcorner R=0$ with $\omega$ given in (2.3). This is easily seen from the explicit form (2.9) of the curvature $R$.

\section{TWISTOR SPACES OF THE SIX-SPHERE}

\section{A. Twistor spaces of $S^{6}$}

We mentioned in the Introduction that one can associate with $S^{6}$ two different twistor spaces, both with an integrable almost complex structure. The larger one, $\operatorname{Tw}\left(S^{6}\right)=\operatorname{Spin}(7) / \mathrm{U}(3)$, belongs to the round sphere $S^{6}=\operatorname{Spin}(7) / \mathrm{SU}(4)$ having the full Lorentz symmetry $\mathrm{SO}(6) \cong \mathrm{SU}(4) / \mathbb{Z}_{2}$ on the tangent spaces and the Levi-Civita connection. The smaller twistor space, $\mathcal{Z}=G_{2} / \mathrm{U}(2)$, is associated with the nearly Kähler coset space $S^{6}=G_{2} / \mathrm{SU}(3)$ having the canonical connection $\Gamma$ with a torsion given in (2.8). The space $\mathcal{Z}$ is a complex submanifold of $\operatorname{Tw}\left(S^{6}\right)$. Note that the Hermitian Yang-Mills Equation (2.11) on $S^{6}$ is $S U(3)$ invariant but not invariant under the full orthogonal group $\mathrm{SO}(6)$. This shows that the reduced twistor space $\mathcal{Z}$ is more suitable than $\operatorname{Tw}\left(S^{6}\right)$ for a description of instantons on $S^{6}$.

\section{B. Coset representation of $\mathbb{C} P^{2}$}

Let us consider the projection

$$
\pi: \quad \mathcal{Z} \longrightarrow S^{6}=G_{2} / \mathrm{SU}(3)
$$

with fibres

$$
\mathbb{C} P^{2}=\mathrm{SU}(3) / \mathrm{U}(2) \text {. }
$$

Let $J_{\mathbb{C} P^{2}}$ be a complex structure on $\mathbb{C} P^{2},\left\{y^{\alpha}\right\}$ homogeneous coordinates on $\mathbb{C} P^{2}$ and

$$
\lambda^{1}=\frac{y^{1}}{y^{3}} \quad \text { and } \quad \lambda^{2}=\frac{y^{2}}{y^{3}}
$$

be local complex coordinates on the patch $\mathcal{U}_{3}=\left\{y^{3} \neq 0\right\} \subset \mathbb{C} P^{2}$.

One can choose as a coset representation of $\mathbb{C} P^{2}$ the matrix

$$
V=\frac{1}{\gamma}\left(\begin{array}{cc}
W & \Lambda \\
-\Lambda^{\dagger} & 1
\end{array}\right):=\frac{1}{\gamma}\left(\begin{array}{ccc}
W_{11} & W_{12} & \lambda^{1} \\
W_{21} & W_{22} & \lambda^{2} \\
-\bar{\lambda}^{\overline{1}} & -\bar{\lambda}^{\overline{2}} & 1
\end{array}\right) \in \mathrm{SU}(3),
$$

where

$$
\gamma^{2}:=1+\Lambda^{\dagger} \Lambda=1+\lambda^{1} \bar{\lambda}^{\overline{1}}+\lambda^{2} \bar{\lambda}^{\overline{2}} \quad \text { and } \quad W=W^{\dagger}=\gamma \cdot \mathbb{1}_{2}-\frac{1}{\gamma+1} \Lambda \Lambda^{\dagger} .
$$


It is a local section of the bundle $\mathrm{SU}(3) \rightarrow \mathbb{C} P^{2}=\mathrm{SU}(3) / \mathrm{U}(2)$. From (3.4) and (3.5), it is easy to see that

$$
W \Lambda=\Lambda \quad \text { and } \quad W^{2}=\gamma^{2}-\Lambda \Lambda^{\dagger} \quad \Leftrightarrow \quad V^{\dagger} V=\mathbb{1}_{3}=V V^{\dagger} .
$$

\section{Flat connection on $\mathcal{Z}$}

Using the group element (3.4) to parametrize the typical $\mathbb{C} P^{2}$-fibre in (3.1), we introduce a flat connection $\hat{\mathcal{A}}_{0}$ on the trivial bundle $G_{2} \times \mathcal{Z} \rightarrow \mathcal{Z}$ as

$$
\hat{\mathcal{A}}_{0}=\hat{V}^{\dagger} \mathcal{A}_{0} \hat{V}+\hat{V}^{\dagger} \mathrm{d} \hat{V} \quad \text { with } \quad \hat{V}=\left(\begin{array}{ccc}
\bar{V} & 0 & 0 \\
0 & 1 & 0 \\
0 & 0 & V
\end{array}\right) \in G_{2}, \quad V \in \operatorname{SU}(3),
$$

where $\mathcal{A}_{0}$ is given in (2.6). One gets

$$
\hat{\mathcal{A}}_{0}=\left(\begin{array}{ccc}
\overline{\hat{\Gamma}} & -\hat{\theta}^{\dagger} & \hat{B} \\
\hat{\theta} & 0 & \overline{\hat{\theta}} \\
\overline{\hat{B}} & -\overline{\hat{\theta}}^{\dagger} & \hat{\Gamma}
\end{array}\right)=\left(\begin{array}{ccc}
\bar{V}^{\dagger} \bar{\Gamma} \bar{V}+\bar{V}^{\dagger} \mathrm{d} \bar{V} & -\bar{V}^{\dagger} \theta^{\dagger} & \bar{V}^{\dagger} B V \\
\theta \bar{V} & 0 & \bar{\theta} V \\
V^{\dagger} \bar{B} \bar{V} & -V^{\dagger} \bar{\theta}^{\dagger} & V^{\dagger} \Gamma V+V^{\dagger} \mathrm{d} V
\end{array}\right),
$$

and for the curvature $\hat{\mathcal{F}}_{0}=\mathrm{d} \hat{\mathcal{A}}_{0}+\hat{\mathcal{A}}_{0} \wedge \hat{\mathcal{A}}_{0}$, we obtain

$$
\hat{\mathcal{F}}_{0}=\left(\begin{array}{ccc}
\overline{\hat{R}}-\hat{\theta}^{\dagger} \wedge \hat{\theta}+\hat{B} \wedge \overline{\hat{B}} & -\left(\mathrm{d} \hat{\theta}^{\dagger}+\overline{\hat{\Gamma}} \wedge \hat{\theta}^{\dagger}+\hat{B} \wedge \overline{\hat{\theta}}^{\dagger}\right) & \mathrm{d} \hat{B}+\overline{\hat{\Gamma}} \wedge \hat{B}+\hat{B} \wedge \hat{\Gamma}-\hat{\theta}^{\dagger} \wedge \overline{\hat{\theta}} \\
\mathrm{d} \hat{\theta}+\hat{\theta} \wedge \hat{\Gamma}+\overline{\hat{\theta}} \wedge \overline{\hat{B}} & -\left(\hat{\theta} \wedge \hat{\theta}^{\dagger}+\overline{\hat{\theta}} \wedge \overline{\hat{\theta}}^{\dagger}\right) & \mathrm{d} \overline{\hat{\theta}}+\overline{\hat{\theta}} \wedge \hat{\Gamma}+\hat{\theta} \wedge \hat{B} \\
\mathrm{~d} \overline{\hat{B}}+\hat{\Gamma} \wedge \hat{\hat{B}}+\overline{\hat{B}} \wedge \hat{\bar{\Gamma}}-\hat{\hat{\theta}}^{\dagger} \wedge \hat{\theta} & -\left(\mathrm{d} \overline{\hat{\theta}}^{\dagger}+\hat{\Gamma} \wedge \overline{\hat{\theta}}^{\dagger}+\hat{\hat{B}} \wedge \hat{\theta}^{\dagger}\right) & \hat{R}-\overline{\hat{\theta}}^{\dagger} \wedge \hat{\theta}+\hat{\hat{B}} \wedge \hat{B}
\end{array}\right) .
$$

\section{Maurer-Cartan equations on $\mathcal{Z}$}

From the flatness $\hat{\mathcal{F}}_{0}=0$ with (3.8) and (3.9), it follows that

$$
\begin{aligned}
& \overline{\hat{\theta}}^{\dagger}=V^{\dagger} \bar{\theta}^{\dagger} \Rightarrow\left(\begin{array}{c}
\hat{\theta}^{1} \\
\hat{\theta}^{2} \\
\hat{\theta}^{3}
\end{array}\right)=\frac{1}{\gamma}\left(\begin{array}{ccc}
W_{11} & W_{12} & -\lambda^{1} \\
W_{21} & W_{22} & -\lambda^{2} \\
\bar{\lambda}^{\overline{1}} & \bar{\lambda}^{2} & 1
\end{array}\right)\left(\begin{array}{c}
\theta^{1} \\
\theta^{2} \\
\theta^{3}
\end{array}\right) \text { and } \\
& \hat{B}=\bar{V}^{\dagger} B V=\sigma\left(\begin{array}{ccc}
0 & \hat{\theta}^{3} & -\hat{\theta}^{2} \\
-\hat{\theta}^{3} & 0 & \hat{\theta}^{1} \\
\hat{\theta}^{2} & -\hat{\theta}^{1} & 0
\end{array}\right),
\end{aligned}
$$

where $\hat{\theta}^{\alpha}$ are (1,0)-forms with respect to $\pi^{*} J \oplus J_{\mathbb{C} P^{2}}$. The latter is not integrable since

$$
\mathrm{d}\left(\begin{array}{c}
\hat{\theta}^{1} \\
\hat{\theta}^{2} \\
\hat{\theta}^{3}
\end{array}\right)+\hat{\Gamma} \wedge\left(\begin{array}{c}
\hat{\theta}^{1} \\
\hat{\theta}^{2} \\
\hat{\theta}^{3}
\end{array}\right)=\frac{2}{\sqrt{3}}\left(\begin{array}{c}
\hat{\theta}^{\overline{2}} \wedge \hat{\theta}^{\overline{3}} \\
\hat{\theta}^{\overline{3}} \wedge \hat{\theta}^{\overline{1}} \\
\hat{\theta}^{\overline{1}} \wedge \hat{\theta}^{\overline{2}}
\end{array}\right) \quad \Leftrightarrow \quad \mathrm{d} \hat{\theta}^{\alpha}+\hat{\Gamma}_{\beta}^{\alpha} \wedge \hat{\theta}^{\beta}=\hat{T}^{\alpha},
$$

and we see a non-vanishing torsion $\hat{T}^{\alpha}$ with $(0,2)$-components. Here, the connection on the tangent bundle $T \mathcal{Z}$ reads

$$
\hat{\Gamma}=V^{\dagger} \Gamma V+V^{\dagger} \mathrm{d} V=\left(\begin{array}{ccc}
C_{11}+b & C_{12} & \hat{\theta}^{4} \\
C_{21} & C_{22}+b & \hat{\theta}^{5} \\
-\hat{\theta}^{\overline{4}} & -\hat{\theta}^{\overline{5}} & -2 b
\end{array}\right)
$$

where

$$
\left(\begin{array}{ll}
C & 0 \\
0 & 0
\end{array}\right)+\left(\begin{array}{cc}
b \cdot \mathbf{1}_{2} & 0 \\
0 & -2 b
\end{array}\right)=\left(\begin{array}{ccc}
C_{11}+b & C_{12} & 0 \\
C_{21} & C_{22}+b & 0 \\
0 & 0 & -2 b
\end{array}\right)
$$


is the canonical $u(2)$-valued connection on the principal bundle $G_{2} \rightarrow G_{2} / \mathrm{U}(2)=\mathcal{Z}$, and $\hat{\theta}^{4}, \hat{\theta}^{5}$ are $(1,0)$-forms on the $\mathbb{C} P^{2}$ fibres of the twistor bundle (3.1).

\section{E. Curvature of the connection $\hat{\Gamma}$}

Consider the curvature $\hat{R}=\mathrm{d} \hat{\Gamma}+\hat{\Gamma} \wedge \hat{\Gamma}$ of the connection $\hat{\Gamma}$ on $T \mathcal{Z}$ given by (3.12) and (3.4). One can easily calculate $\hat{R}$ from (3.9) and obtain

$$
\begin{aligned}
\hat{R} & =\left(\begin{array}{ccc}
F_{11}^{C}+\mathrm{d} b-\hat{\theta}^{4 \overline{4}} & F_{12}^{C}-\hat{\theta}^{4 \overline{5}} & \mathrm{~d} \hat{\theta}^{4}+\left(C_{11}+3 b\right) \wedge \hat{\theta}^{4}+C_{12} \wedge \hat{\theta}^{5} \\
F_{21}^{C}-\hat{\theta}^{5 \overline{4}} & F_{22}^{C}+\mathrm{d} b-\hat{\theta}^{5 \overline{5}} & \mathrm{~d} \hat{\theta}^{5}+C_{21} \wedge \hat{\theta}^{4}+\left(C_{22}+3 b\right) \wedge \hat{\theta}^{5} \\
-\left(\mathrm{d} \hat{\theta}^{\overline{4}}+\hat{\theta}^{\overline{4}} \wedge\left(C_{11}+3 b\right)+\hat{\theta}^{\overline{5}} \wedge C_{21}\right) & -\left(\mathrm{d} \hat{\theta}^{\overline{5}}+\hat{\theta}^{\overline{4}} \wedge C_{12}+\hat{\theta}^{\overline{5}} \wedge\left(C_{22}+3 b\right)\right) & -2 \mathrm{~d} b+\hat{\theta}^{4 \overline{4}}+\hat{\theta}^{5 \overline{5}}
\end{array}\right), \\
& =\left(\begin{array}{ccc}
\frac{1}{3}\left(2 \hat{\theta}^{1 \overline{1}}-\hat{\theta}^{2 \overline{2}}-\hat{\theta}^{3 \overline{3}}\right) & \hat{\theta}^{1 \overline{2}} & \hat{\theta}^{1 \overline{3}} \\
\hat{\theta}^{2 \overline{1}} & \frac{1}{3}\left(-\hat{\theta}^{1 \overline{1}}+2 \hat{\theta}^{2 \overline{2}}-\hat{\theta}^{3 \overline{3}}\right) & \hat{\theta}^{2 \overline{3}} \\
\hat{\theta}^{3 \overline{1}} & \hat{\theta}^{3 \bar{z}} & \frac{1}{3}\left(-\hat{\theta}^{1 \overline{1}}-\hat{\theta}^{2 \bar{z}}+2 \hat{\theta}^{3 \overline{3}}\right)
\end{array}\right)
\end{aligned}
$$

where

$$
F^{C}=\mathrm{d} C+C \wedge C=\left(\begin{array}{ll}
F_{11}^{C} & F_{12}^{C} \\
F_{21}^{C} & F_{22}^{C}
\end{array}\right) \in \operatorname{su}(2) .
$$

The components of $F^{C}$ and $\mathrm{d} b$ can be read off from (3.14). Equation (3.14) also tells us that

$$
\mathrm{d}\left(\begin{array}{c}
\hat{\theta}^{4} \\
\hat{\theta}^{5}
\end{array}\right)+\left(C+3 b \cdot \mathbb{1}_{2}\right) \wedge\left(\begin{array}{c}
\hat{\theta}^{4} \\
\hat{\theta}^{5}
\end{array}\right)=\left(\begin{array}{c}
\hat{\theta}^{1 \overline{3}} \\
\hat{\theta}^{2 \overline{3}}
\end{array}\right) .
$$

Together with (3.11), this can be considered as the Maurer-Cartan equations on $\mathcal{Z}$ for the forms $\hat{\theta}^{A}$, $A=1, \ldots, 5$. Those are (1,0)-forms with respect to an almost complex structure $\mathcal{J}_{-}=\pi^{*} J \oplus J_{\mathbb{C} P^{2}}$ on $\mathcal{Z}$ defined via

$$
\mathcal{J}_{-} \hat{\theta}^{A}=\mathrm{i} \hat{\theta}^{A} .
$$

The non-vanishing $(0,2)$-type components of the torsion $\hat{T}^{A}$ obstruct the integrability of $\mathcal{J}_{-}$.

\section{F. Integrable almost complex structure on $\mathcal{Z}$}

We may introduce a different almost complex structure $\mathcal{J}_{+}$on $\mathcal{Z}$ with the property

$$
\mathcal{J}_{+} \vartheta^{A}=\mathrm{i} \vartheta^{A} \quad \text { for } \quad \vartheta^{1}:=\hat{\theta}^{1}, \quad \vartheta^{2}:=\hat{\theta}^{2}, \quad \vartheta^{3}:=\hat{\theta}^{\overline{3}}, \quad \vartheta^{4}:=\hat{\theta}^{4} \quad \text { and } \vartheta^{5}:=\hat{\theta}^{5}
$$

and denote $\overline{\vartheta^{A}}=: \vartheta^{\bar{A}}$. Then from (3.11) and (3.16), we obtain

$$
\mathrm{d} \vartheta^{A}+\widetilde{\Gamma}_{B}^{A} \wedge \vartheta^{B}=\widetilde{T}^{A},
$$

where the connection $\widetilde{\Gamma}=\left(\widetilde{\Gamma}_{B}^{A}\right)$ and the torsion $\widetilde{T}=\left(\widetilde{T}^{A}\right)$ are given by

$$
\widetilde{\Gamma}=\left(\begin{array}{ccccc}
C_{11}+b & C_{12} & 0 & 0 & 0 \\
C_{21} & C_{22}+b & 0 & 0 & 0 \\
0 & 0 & 2 b & 0 & 0 \\
0 & 0 & 0 & C_{11}+3 b & C_{12} \\
0 & 0 & 0 & C_{21} & C_{22}+3 b
\end{array}\right) \text { and } \tilde{T}=\left(\begin{array}{c}
\frac{2}{\sqrt{3}} \vartheta^{3 \overline{2}}-\vartheta^{4 \overline{3}} \\
-\frac{2}{\sqrt{3}} \vartheta^{3 \overline{1}}-\vartheta^{5 \overline{3}} \\
-\frac{2}{\sqrt{3}} \vartheta^{12}+\vartheta^{4 \overline{1}}+\vartheta^{5 \overline{2}} \\
\vartheta^{13} \\
\vartheta^{23}
\end{array}\right)
$$

Note that $\widetilde{\Gamma}$ is the canonical $u(2)$-valued connection on the tangent bundle $T \mathcal{Z}$, and $\widetilde{T}$ is the torsion of $\widetilde{\Gamma}$. The torsion $\widetilde{T}^{A}$ in (3.19) has no (0,2)-components with respect to the almost complex structure $\mathcal{J}_{+}$. Therefore, $\mathcal{J}_{+}$is integrable, i.e., $\left(\mathcal{Z}, \mathcal{J}_{+}\right)$is a complex manifold. 


\section{TWISTOR DESCRIPTION OF INSTANTON BUNDLES OVER $\boldsymbol{S}^{6}$}

\section{A. Pulled-back curvature}

Consider a complex vector bundle $E$ over $S^{6}$ with a connection one-form $\mathcal{A}$ having curvature $\mathcal{F}$. Recall that $(E, \mathcal{A})$ is called an instanton bundle if $\mathcal{A}$ satisfies the Hermitian Yang-Mills (HYM or DUY) Equation (2.11), which on $S^{6}$ can be written in the form

$$
\begin{array}{ccc}
\mathcal{F}^{0,2}=0 & \Leftrightarrow & \Omega \wedge \mathcal{F}=0, \\
\omega\lrcorner \mathcal{F}=0 & \Leftrightarrow \quad \omega \wedge \omega \wedge \mathcal{F}=0 .
\end{array}
$$

Here, $(\omega, \Omega)$ given in (2.3) are forms defining on $S^{6}$ a nearly Kähler structure. Note that, in this case, (4.2) follows from (4.1) due to (2.10).

Consider the twistor fibration (3.1). Let $(\widetilde{\mathcal{E}}, \widetilde{\mathcal{A}})=\left(\pi^{*} E, \pi^{*} \mathcal{A}\right)$ be the pulled-back instanton bundle over $\mathcal{Z}$ with curvature $\widetilde{\mathcal{F}}=\mathrm{d} \widetilde{\mathcal{A}}+\widetilde{\mathcal{A}} \wedge \widetilde{\mathcal{A}}$. We have

$$
\widetilde{\mathcal{F}}=\frac{1}{2} \widetilde{\mathcal{F}}_{\alpha \beta} \vartheta^{\alpha} \wedge \vartheta^{\beta}+\widetilde{\mathcal{F}}_{\alpha \bar{\beta}} \vartheta^{\alpha} \wedge \vartheta^{\bar{\beta}}+\frac{1}{2} \widetilde{\mathcal{F}}_{\bar{\alpha} \bar{\beta}} \vartheta^{\bar{\alpha}} \wedge \vartheta^{\bar{\beta}}=\pi^{*} \mathcal{F} .
$$

Using the relation (3.10) between $\theta^{\alpha}$ and $\hat{\theta}^{\alpha}$ as well as the definition (3.18) of $\vartheta^{A}$, we obtain

$$
\widetilde{\mathcal{F}}_{\overline{1} \overline{2}}=\frac{1}{\gamma}\left\{\mathcal{F}_{\overline{1} \overline{2}}+\lambda^{1} \mathcal{F}_{\overline{2} \overline{3}}+\lambda^{2} \mathcal{F}_{\overline{3} \overline{1}}\right\} .
$$

Vanishing of $\widetilde{\mathcal{F}}_{\overline{1} \overline{2}}$ for all values of $\left(\lambda^{1}, \lambda^{2}\right) \in \mathbb{C} P^{2}$ is equivalent to the instanton Eqs. (4.1) and (4.2). In contrast, for $\widetilde{\mathcal{F}}_{\overline{1} \overline{3}}$ and $\widetilde{\mathcal{F}}_{\overline{2} \overline{3}}$, we obtain complicated expressions, which vanish for all $\lambda^{1}, \lambda^{2}$ only if all components of the curvature $\mathcal{F}$ vanish. This yields the trivial case of a flat connection on $E$. In homogeneous coordinates $y^{\alpha}$ on $\mathbb{C} P^{2}$, this condition can be written as

$$
\widetilde{\mathcal{F}}_{\overline{1} \overline{2}}=0 \quad \Leftrightarrow \quad y^{\alpha} \varepsilon_{\alpha \beta \gamma} \mathcal{F}^{\beta \gamma}=0,
$$

where the indices $\bar{\alpha}, \bar{\beta}, \ldots$ are raised with the metric $\delta^{\alpha \bar{\beta}}$.

\section{B. Correspondence of bundles}

Let us denote by $L_{A}$ vector fields on $\mathcal{Z}$ of type $(1,0)$ (with respect to the complex structure $\mathcal{J}_{+}$) and by $L_{\bar{A}}$ their complex conjugates, $A=1, \ldots, 5$. Then we can introduce a rank-2 subbundle ${ }^{41} \mathcal{T}_{(2)}^{0,1}$ of $T^{0,1} \mathcal{Z}$ spanned by $L_{\overline{1}}$ and $L_{\overline{2}}$ as well as a rank-4 subbundle $\mathcal{T}_{(4)}^{0,1}$ of $T^{0,1} \mathcal{Z}$ with $\left\{L_{\overline{1}}, L_{\overline{2}}, L_{\overline{4}}, L_{\overline{5}}\right\}$ as a basis. Note that $\widetilde{\mathcal{F}}_{\overline{1} \overline{2}}$ is the curvature of a partial connection $\nabla_{\mathcal{T}_{(2)}^{0,1}}$ (see Ref. 33) along the distribution $\mathcal{T}_{(2)}^{0,1}$, and we can extend it to a partial connection $\nabla_{\mathcal{T}_{(4)}^{0,1}}$ along $\mathcal{T}_{(4)}^{0,1}$ by putting $\widetilde{\mathcal{A}}_{\overline{4}}=$ $0=\widetilde{\mathcal{A}}_{\overline{5}}$. Neither $\mathcal{T}_{(2)}^{0,1}$, nor $\mathcal{T}_{(4)}^{0,1}$ is integrable as a subbundle of $T^{0,1} \mathcal{Z}$ and, therefore, we cannot consider $\pi^{*} E$ as a Cauchy-Riemann (CR) bundle. This follows from the explicit form of the torsion (3.19) of the $\mathrm{U}(2)$-structure on $\mathcal{Z}$. It would be interesting to repeat our analysis for the other three known homogeneous nearly Kähler spaces and to check whether there exist special cases where the integrability obstructions vanish. However, this is beyond the scope of our paper, which deals with $S^{6}$ only.

In summary, we have the following picture:

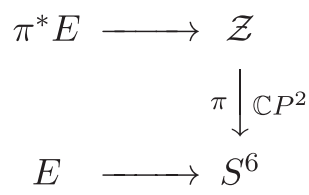

where $(E, \nabla)$ with $\nabla=\mathrm{d}+\mathcal{A}$ is a Hermitian Yang-Mills bundle with curvature $\mathcal{F}=\nabla^{2}$ satisfying (4.1) and (4.2). From the above discussion, we obtain the equivalence of two assertions:

(i) $\quad\left(\pi^{*} E, \pi^{*} \nabla\right)$ has its curvature $\widetilde{\mathcal{F}}=\pi^{*} \mathcal{F}$ vanishing along the distribution $\mathcal{T}_{(4)}^{0,1} \subset T^{0,1} \mathcal{Z}$. 
(ii) $(E, \mathcal{A})$ is a HYM (instanton) bundle over $S^{6}$.

The non-integrability of the distribution $\mathcal{T}_{(4)}^{0,1}$ means that the HYM equations on $S^{6}$ are not integrable, contrary to the self-dual Yang-Mills equations on $S^{4}$. Hence, constructing instanton configurations in six dimensions is a task more complicated than one might expect.

\section{Relation with instantons on $\mathbb{R}^{7}$}

Note that the cone $C\left(S^{6}\right)$ over $S^{6}$ with the metric

$$
\mathrm{d} s_{7}^{2}=\mathrm{d} r^{2}+r^{2} \mathrm{~d} s_{S^{6}}^{2} \quad \text { with } \quad r \in \mathbb{R}_{+}
$$

is flat space, $C\left(S^{6}\right)=\mathbb{R}^{7} \backslash\{0\}$, for a proper normalization of the $S^{6}$ coframe $\left\{e^{a}\right\}$ such that $\rho=1$ in (2.10). Employing the forms $(\omega, \Omega)$ defining the nearly Kähler structure on $S^{6}$, we introduce on $\mathbb{R}^{7}$ the 3 -form

$$
\psi:=r^{2} \omega \wedge \mathrm{d} r+r^{3} \operatorname{Im} \Omega .
$$

It is not difficult to show that (up to index permutation) its only nonzero coefficients are

$$
\psi_{\hat{a} \hat{b} \hat{c}}=1 \quad \text { for } \quad(\hat{a} \hat{b} \hat{c})=(136),(426),(145),(235),(127),(347),(567)
$$

in the basis $\left\{\mathrm{d} x^{\hat{a}}\right\}$ with coordinates $x^{\hat{a}}$ on $\mathbb{R}^{7}$ such that $\delta_{\hat{a} \hat{b}} x^{\hat{a}} x^{\hat{b}}=r^{2}$. The above 3 -form $\psi$ defines a $G_{2}$-structure on $\mathbb{R}^{7}$, i.e., it is invariant under the $G_{2} \subset \mathrm{SO}(7)$ action. Its components (4.9) are often called octonionic structure constants.

Consider now a complex vector bundle $\mathcal{E}$ over $\mathbb{R}^{7}$ with a connection $\mathcal{A}^{\prime}$ and curvature $\mathcal{F}^{\prime}$. Employing the Hodge operator $*$ in $\mathbb{R}^{7}$, we impose on $\mathcal{A}^{\prime}$ the first-order differential equations

$$
* \psi \wedge \mathcal{F}^{\prime}=0 \quad \Leftrightarrow \quad \psi_{\hat{a} \hat{b} \hat{c}} \mathcal{F}^{\prime} \hat{b} \hat{c}=0,
$$

which are called $G_{2}$-instanton equations. ${ }^{25}$ Their solutions automatically satisfy the Yang-Mills equations on $\mathbb{R}^{7}$.

It was shown by $\operatorname{Tian}^{26}$ that solutions $\mathcal{A}^{\prime}$ of (4.10) obeying also

$$
\left.\left.\partial_{r}\right\lrcorner \mathcal{A}^{\prime}=0 \quad \text { and } \quad \partial_{r}\right\lrcorner \mathcal{F}^{\prime}=0
$$

are equivalent to solutions $\mathcal{A}$ of the HYM Equations (4.1) and (4.2) on $S^{6}$. He calls such configurations tangent instantons on $\mathbb{R}^{7}$. Examples of such instanton solutions were discussed in Ref. 29 and 37.

A twistor description of solutions to (4.10) on any 7-dimensional Riemannian manifold $X$ with $G_{2}$-holonomy was recently proposed by Verbitsky. ${ }^{18}$ Namely, he introduced a so-called CR twistor space of $X$ as the bundle $\pi: S^{6} X \rightarrow X$ of unit six-spheres in the tangent bundle $T X$. For $\mathbb{R}^{7}$, this space is a direct product manifold

$$
\operatorname{Tw}\left(\mathbb{R}^{7}\right)=\mathbb{R}^{7} \times S^{6} .
$$

It was $\operatorname{shown}^{18}$ that the complexified tangent bundle of $\operatorname{Tw}(X)$ has an integrable complex rank-3 subbundle $\mathcal{T}_{(3)}^{0,1}$ if $X$ is a $G_{2}$-holonomy manifold. For a bundle $\mathcal{E}$ over $X$ with a connection $\mathcal{A}^{\prime}$, one can introduce the pulled-back bundle $\left(\pi^{*} \mathcal{E}, \pi^{*} \mathcal{A}^{\prime}\right)$ over $\operatorname{Tw}(X)$. It was proven that $G_{2}$-instanton bundles over $X$ correspond to CR-bundles over $\operatorname{Tw}(X)$ with a flat partial $(0,1)$-connection $\bar{\partial}_{\pi^{*} \mathcal{E}}$ defined on the distribution $\mathcal{T}_{(3)}^{0,1}{ }^{18}$ In other words, the $G_{2}$-instanton equations on $X$ are equivalent to the equations $\bar{\partial}_{\pi^{*} \mathcal{E}}^{2}=0$ on $\operatorname{Tw}(X)$. This theorem obviously applies to the case of $X=\mathbb{R}^{7}$. Specializing then to tangent solutions (in the sense of (4.11)) to the $G_{2}$-instanton Equation (4.10) on $\mathbb{R}^{7}$ will yield solutions of the HYM equations on $S^{6}$. Thus, the twistor description of instantons on $S^{6}$ is related to the twistor description of $G_{2}$-instanton solutions on $\mathbb{R}^{7}$.

\section{ACKNOWLEDGMENTS}

We would like to thank Vicente Cortés, Klaus Hulek, and Misha Verbitsky for useful discussions in the framework of the Graduiertenkolleg "Analysis, Geometry and String Theory." This work was 
in part supported by the Deutsche Forschungsgemeinschaft and the Riemann Center for Geometry and Physics of Leibniz Universität Hannover.

${ }^{1}$ L. P. Hughston, "Applications of Cartan spinors to differential geometry in higher dimensions," in Spinors in Physics and Geometry, edited by G. Furlan and A. Trautman (World Scientific, 1987).

${ }^{2}$ C. Saemann and M. Wolf, "On twistors and conformal field theories from six dimensions"; e-print arXiv:1111.2539 [hep-th].

${ }^{3}$ L. J. Mason, R. A. Reid-Edwards, and A. Taghavi-Chabert, "Conformal field theories in six-dimensional twistor space," J. Geom. Phys. 62, 2353 (2012); e-print arXiv:1111.2585 [hep-th].

${ }^{4}$ C. Saemann and M. Wolf, "Non-Abelian tensor multiplet equations from twistor space"; e-print arXiv:1205.3108 [hep-th].

${ }^{5}$ C. Saemann, R. Wimmer, and M. Wolf, "A twistor description of six-dimensional $\mathcal{N}=(1,1)$ super Yang-Mills theory," J. High Energy Phys. 05 (2012) 020; e-print arXiv:1201.6285 [hep-th].

${ }^{6}$ C. Cheung and D. O'Connell, "Amplitudes and spinor-helicity in six dimensions," J. High Energy Phys. 07 (2009) 075; e-print arXiv:0902.0981 [hep-th]; T. Dennen, Y.-T. Huang, and W. Siegel, "Supertwistor space for 6d maximal super YangMills," J. High Energy Phys. 04 (2010) 127; e-print arXiv:0910.2688 [hep-th]; A. Brandhuber, D. Korres, D. Koschade, and G. Travaglini, "One-loop amplitudes in six-dimensional $(1,1)$ theories from generalised unitarity," J. High Energy Phys. 02 (2011) 077; e-print arXiv: 1010.1515 [hep-th].

${ }^{7}$ A. A. Belavin, A. M. Polyakov, A. S. Schwartz, and Yu. S. Tyupkin, "Pseudoparticle solutions of the Yang-Mills equations," Phys. Lett. B 59, 85 (1975).

${ }^{8}$ R. S. Ward, "On self-dual gauge fields,” Phys. Lett. A 61, 81 (1977).

${ }^{9}$ M. F. Atiyah, N. J. Hitchin, and I. M. Singer, "Self-duality in four-dimensional Riemannian geometry," Proc. R. Soc. London, Ser. A 362, 425 (1978).

${ }^{10}$ M. F. Atiyah, N. J. Hitchin, V. G. Drinfeld, and Yu. I. Manin, “Construction of instantons," Phys. Lett. A 65, 185 (1978).

${ }^{11}$ L. B. Bergery and T. Ochiai, "On some generalization of the construction of twistor spaces," in: Global Riemannian Geometry, edited by T. J. Willmore and N. J. Hitchin (Ellis Horwood, 1984); N. R. O'Brian and J. H. Rawnsley, "Twistor spaces," Ann. Global Anal. Geom. 3, 29 (1985); F. E. Burstall, "Riemannian twistor spaces and holonomy groups," in Twistors in Mathematics and Physics, L. M. S. Lecture Notes Vol. 156, edited by T. N. Bailey and R. J. Baston (Cambridge University Press, 1990).

${ }^{12}$ R. L. Bryant, "Submanifolds and special structures on the octonians," J. Diff. Geom. 17, 185 (1982); "Lie groups and twistor spaces," Duke Math. J. 52, 223 (1985).

${ }^{13}$ S. Salamon, "Harmonic and holomorphic maps," Lect. Notes Math. 1164, 161 (1985).

${ }^{14}$ E. Musso, "On the twistor space of the six-sphere," Bull. Aust. Math. Soc. 39, 119 (1989).

${ }^{15}$ D. V. Alekseevsky and M. M. Graev, "G-structures of twistor type and their twistor spaces," J. Geom. Phys. 3, 203 (1993).

${ }^{16}$ J.-B. Butruille, "Twistors and 3-symmetric spaces," Proc. London Math. Soc. 96, 738 (2008); e-print arXiv:math/0604394 [math.DG].

${ }^{17}$ L. Borisov, S. Salamon, and J. Viaclovsky, "Twistor geometry and warped product orthogonal complex structures," Duke Math. J. 156, 125 (2011); e-print arXiv:0905.3662 [math.DG].

${ }^{18}$ M. Verbitsky, “A CR twistor space of a $G_{2}$-manifold," Diff. Geom. Appl. 29, 101 (2011); e-print arXiv:1003.3170 [math.DG].

${ }^{19}$ M. Wolf, "Contact manifolds, contact instantons, and twistor geometry," J.High Energy Phys. 07, 074 (2012); e-print arXiv: 1203.3423 [hep-th].

${ }^{20}$ E. Corrigan, C. Devchand, D. B. Fairlie, and J. Nuyts, "First order equations for gauge fields in spaces of dimension greater than four," Nucl. Phys. B 214, 452 (1983); R. S. Ward, "Completely solvable gauge field equations in dimension greater than four," Nucl. Phys. B 236, 381 (1984).

${ }^{21}$ S. K. Donaldson, "Anti-self-dual Yang-Mills connections on a complex algebraic surface and stable vector bundles," Proc. London Math. Soc. 50, 1 (1985); S. K. Donaldson, "Infinite determinants, stable bundles and curvature," Duke Math. J. 54, 231 (1987); K. K. Uhlenbeck and S.-T. Yau, "On the existence of Hermitian-Yang-Mills connections on stable bundles over compact Kähler manifolds,” Commun. Pure Appl. Math. 39, 257 (1986).

${ }^{22}$ M. M. Capria, and S. M. Salamon, "Yang-Mills fields on quaternionic spaces," Nonlinearity 1, 517 (1988); R. R. Carrión, "A generalization of the notion of instanton," Diff. Geom. Applic. 8, 1 (1998).

${ }^{23}$ T. A. Ivanova and A. D. Popov, "(Anti)self-dual gauge fields in dimension $d \geq 4$," Theor. Math. Phys. 94, 225 (1993).

${ }^{24}$ L. Baulieu, H. Kanno, and I. M. Singer, "Special quantum field theories in eight and other dimensions," Commun. Math. Phys. 194, 149 (1998); e-print arXiv:hep-th/9704167; M. Blau and G. Thompson, "Euclidean SYM theories by time reduction and special holonomy manifolds," Phys. Lett. B 415, 242 (1997); e-print arXiv:hep-th/9706225; B. S. Acharya, J. M. Figueroa-O'Farrill, B. J. Spence, and M. O'Loughlin, "Euclidean D-branes and higher-dimensional gauge theory," Nucl. Phys. B 514, 583 (1998); e-print arXiv:hep-th/9707118.

${ }^{25}$ S. K. Donaldson and R. P. Thomas, "Gauge theory in higher dimensions," in The Geometric Universe, edited by S. A. Huggett et al. (Oxford University Press, Oxford, 1998); S. K. Donaldson and E. Segal, "Gauge theory in higher dimensions II," in Surveys in Differential Geometry Vol. 16, edited by N. C. Leung and S.-T. Yau (International, Boston, 2011); e-print arXiv:0902.3239 [math.DG].

${ }^{26}$ G. Tian, "Gauge theory and calibrated geometry," Ann. Math. 151, 193 (2000); e-print arXiv:math/0010015 [math.DG].

${ }^{27} \mathrm{M}$. Wolf, "A connection between twistors and superstring sigma models on coset superspaces," J. High Energy Phys. 09 (2009) 071; e-print arXiv:0907.3862 [hep-th].

${ }^{28}$ A. D. Popov, "Non-Abelian vortices, super-Yang-Mills theory and Spin(7)-instantons," Lett. Math. Phys. 92 (2010) 253; e-print arXiv:0908.3055 [hep-th]; A. D. Popov and R. J. Szabo, "Double quiver gauge theory and nearly Kähler flux compactifications,” J. High Energy Phys. 02 (2012) 033; e-print arXiv:1009.3208 [hep-th]. 
${ }^{29}$ D. Harland and C. Nölle, "Instantons and killing spinors," J. High Energy Phys. 03 (2012) 082; e-print arXiv:1109.3552 [hep-th]; T. A. Ivanova and A. D. Popov, "Instantons on special holonomy manifolds," Phys. Rev. D 85, 105012 (2012); e-print arXiv: 1203.2657 [hep-th].

${ }^{30}$ R. Penrose, "Non-linear gravitons and curved twistor theory," Gen. Rel. Grav. 7, 31 (1976).

${ }^{31}$ Yu. I. Manin and H. Le Minh, "The Radon-Penrose transformation for the group SO(8), and instantons," Math. USSR, Izv. 28, 189 (1987).

${ }^{32}$ R. L. Bryant, "Remarks on the geometry of almost complex 6-manifolds," Asian J. Math. 10, 561 (2006); e-print arXiv:math/0508428 [math.DG].

${ }^{33}$ J. H. Rawnsley, "Flat partial connections and holomorphic structures in $\mathbb{C}^{\infty}$ vector bundles," Proc. Am. Math. Soc. 73, 391 (1979).

${ }^{34}$ A. D. Popov, C. Saemann, and M. Wolf, "The topological B-model on a mini-supertwistor space and supersymmetric Bogomolny monopole equations," J. High Energy Phys. 10 (2005) 058; e-print arXiv:hep-th/0505161.

${ }^{35}$ A. J. Macfarline, “The sphere $S^{6}$ viewed as a $G_{2} / \mathrm{SU}(3)$ coset space," Int. J. Mod. Phys. A 17, 2595 (2002).

${ }^{36} \mathrm{~S}$. Chiossi and S. Salamon, "The intrinsic torsion of SU(3) and $G_{2}$ structures," in Differential Geometry, Valencia, 2001, edited by O. Gil-Medrano and V. Miquel (World Scientific, River Edge, NJ, 2002); e-print arXiv:math/0202282 [math-dg].

${ }^{37}$ D. Harland, T. A. Ivanova, O. Lechtenfeld, and A. D. Popov, "Yang-Mills flows on nearly Kähler manifolds and $G_{2}$ instantons," Commun. Math. Phys. 300, 185 (2010); e-print arXiv:0909.2730 [hep-th]; K.-P. Gemmer, O. Lechtenfeld, C. Nölle, and A. D. Popov, "Yang-Mills instantons on cones and sine-cones over nearly Kähler manifolds," J. High Energy Phys. 09 (2011) 103; e-print arXiv:1108.3951 [hep-th].

${ }^{38}$ Instead of $S^{6}$, one can also consider $\mathbb{R}^{6}=S^{6} \backslash\{\infty\}$ with a metric twistor space $Q_{6}^{\prime}=\operatorname{Tw}\left(\mathbb{R}^{6}\right) \subset Q_{6}$ as an intersection of the quadric $Q_{6}$ with $\mathbb{C} P^{7} \backslash \mathbb{C} P^{3}$. This space $Q_{6}^{\prime}$ is also the twistor space for the complexified space-time $\mathbb{C}^{6}$ with a double fibration establishing the correspondence between subspaces in $Q_{6}^{\prime}$ and $\mathbb{C}^{6}$ (see, e.g., Refs. 2 and 3 and references therein). There is no such correspondence in the real framework where the twistor space is the bundle of almost complex structures on a manifold.

${ }^{39}$ In the mathematical literature, they are often called Hermitian Yang-Mills equations.

${ }^{40}$ From a bundle with curvature $\mathcal{F}$ of non-zero degree, we can obtain a zero-degree bundle $E$ by considering $\widetilde{\mathcal{F}}=\mathcal{F}$ $-\frac{1}{k}(\operatorname{tr} \mathcal{F}) \cdot \mathbb{1}_{k}$, where $k=\operatorname{rank} E$.

${ }^{41}$ Recall that $T^{0,1} \mathcal{Z}$ is an integrable subbundle of the complexification of $T \mathcal{Z}$. 